\title{
A nephrologist should be consulted in all cases of acute kidney injury in the ICU: yes
}

Hans Flaatten ${ }^{{ }^{*}(\mathbb{D} \text { and Michael Darmon }}{ }^{2,3}$

(C) 2017 Springer-Verlag Berlin Heidelberg and ESICM

"No man is an island". John Donne (1572-1631)

Acute kidney injury (AKI) is increasingly common [1] and is associated with negative short-term outcome partly as consequences of distant organ-kidney interactions [2]. More strikingly, even mild AKI is related to long-term mortality in ICU survivors [3], increased risk of chronic and end-stage kidney diseases [4], and increased risk of major cardiovascular events [5]. Although risk seems to be correlated with severity and duration of AKI, even mild and transient AKI is associated with higher mortality and morbidity $[3,6]$. Data from ICU survivors suggest that nephron loss occurs following AKI, leading to long-term renal dysfunction: the more severe the AKI is, the more severe the renal sequela is [7]. In this regard, the risk of chronic kidney disease (CKD) and end-stage CKD has been described to be increased by ninefold and fourfold, respectively [4]. Despite the increasing number of studies in this field, the exact epidemiology of renal function following ICU stay remains blurry mainly as a result of the unavoidable number of patients with unknown baseline renal function, the lack of consensus to define renal recovery, and time-dependency of the renal recovery or progression toward CKD [8]. Patients who died will never develop CKD, a limitation not often taken into account in available reports [8]. Furthermore, renal function as assessed by serum creatinine at ICU discharge is unreliable and impaired by sarcopenia [9]. Finally, the impact of subclinical AKI on subsequent nephron loss and risk of CKD

\footnotetext{
*Correspondence: hans.flaatten@uib.no

${ }^{1}$ General ICU, KSK Haukeland University Hospital and Department of Clinical Medicine, University of Bergen, Bergen, Norway Full author information is available at the end of the article
}

For contrasting viewpoints, please go to doi:10.1007/s00134-017-4712-5 and doi:10.1007/s00134-017-4788-y. is unknown and frequently unmentioned. In view of this evidence, and to adequately assess renal history and deal with remaining uncertainties, a systematic follow-up by a nephrologist with adequate renal function assessment may be required.

Beyond research, early and prolonged follow-up by a nephrologist in ICU patients with AKI is mostly theoretical or supported by evidence with a high risk of bias. Although the bulk of ICU-acquired AKI is believed to be multifactorial, specific causes of AKI are often seen in ICU patients and may require specific diagnostic testing or management. Some of these aetiologies, such as postinfectious glomerulonephritis or acute interstitial nephritis, are probably underestimated, and assistance from a nephrologist should be sought more frequently. The benefit from such early nephrologist involvement has been suggested by low level evidence studies [10]. In some countries, their help might also be required to select an adequate renal replacement modality and run intermittent haemodialysis, the latter limiting need for anticoagulation, limiting costs and performing better in subgroups of patients without shock $[11,12]$. Finally, they may participate in ICU multidisciplinary daily rounds, increasing quality and safety as suggested by guidelines from the European Society of Intensive Care Medicine [13]. Later during the ICU stay, at the time of ICU or hospital discharge, their help might be required in assessing residual function, identifying high-risk patients and selecting subgroups of patients in whom renal recovery might deserve to be more clearly assessed. Finally, a nephrologist will obviously be the best-suited clinician to arrange for a proper follow-up, most conveniently through an outpatient nephrology clinic. Although input of a nephrologist is to a large extent speculative, there is some evidence that suggests an improved survival rate associated with de novo follow-up by a nephrologist in survivors of AKI [14].

\section{Springer}




\section{Why hesitate?}

First, because of the remaining uncertainty regarding validity and the magnitude of the practical benefits. However, in closed ICU models prevailing in European countries, we did not wait for randomized controlled trials to involve surgeons, cardiologists or infectious disease physicians when needed. The increasing and remarkable incidence of acute kidney injury is another obvious limit, and nephrologists may not be able to follow all AKI patients. However, this point, although valid, certainly needs to be tempered down by the $40 \%$ mortality observed at 1 year in patients with moderate to severe AKI [6]. Finally, there is concern about the costs associated with nephrology follow-up. An important limitation of a systematic follow-up by a nephrologist will also be dictated by the healthcare system priorities and sustainability of this strategy. All these limits are valid and may be adequate counterarguments.

However, debating whether nephrology consultation in AKI patients may be required may be perceived as intentional blindness toward current evidence:

- Acute kidney injury is an important issue from the point of view of the patient as well as the healthcare system.

- Long-term consequences of AKI are frequently underestimated and follow-up of AKI patients may certainly be improved.

- We are in urgent need of pragmatic and efficient strategies limiting both short-term and long-term consequences of AKI.

- Early and prolonged nephrology follow-up is a promising non-pharmacological strategy that may increase long-term survival and limit progression toward chronic kidney diseases.

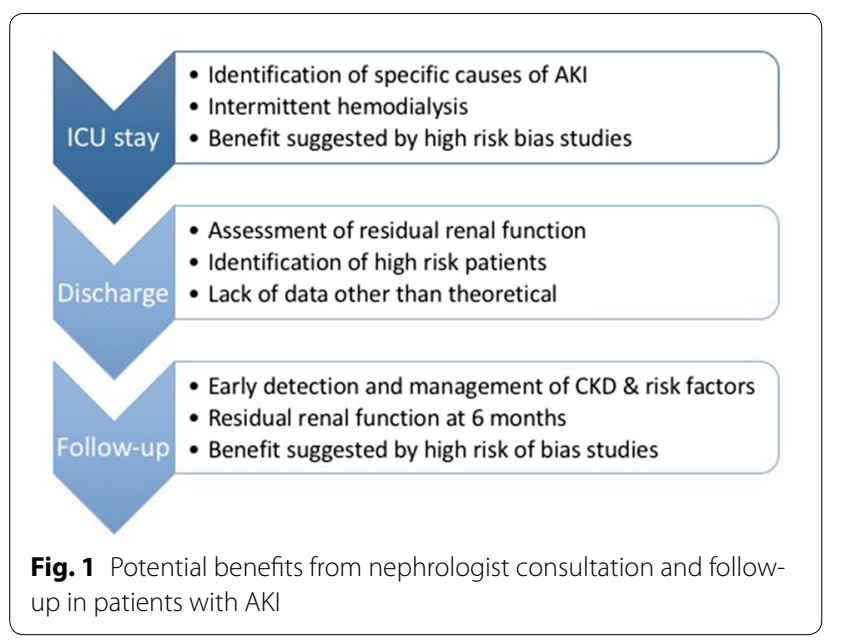

Even with previously mentioned limits in mind, it is time to advocate an early consultation with further systematic follow-up by a nephrologist as the current best solution (Fig. 1). That said, trials assessing overall benefits of such follow-up are urgently needed. Such studies may help to stratify risks, evaluating costs and validating the strategy also in the light of patient-centred outcome and distributive justice. In the meantime, long-term followup by intensivists or general practitioners is certainly warranted. At hospital discharge planning further evaluation in patients having an episode of AKI is necessary. This should at least include a serum creatinine measurement 6 months to 1 year after the ICU stay [15].

\section{Author details \\ ${ }^{1}$ General ICU, KSK Haukeland University Hospital and Department of Clinical Medicine, University of Bergen, Bergen, Norway. ${ }^{2}$ Medical-Surgical ICU, Saint- Etienne University Hospital, Saint-Etienne, France. ${ }^{3}$ Jacques Lisfranc Medical School, Jean Monnet University, Saint-Etienne, France.}

\section{Compliance with ethical standards}

\section{Conflicts of interest}

The authors both declare no conflict of interest.

Received: 9 March 2017 Accepted: 31 March 2017

Published online: 22 May 2017

\section{References}

1. Hsu RK, McCulloch CE, Dudley RA et al (2013) Temporal changes in incidence of dialysis-requiring AKI. J Am Soc Nephrol 24:37-42. doi:10.1681/ ASN.2012080800

2. Hoste EAJ, Bagshaw SM, Bellomo R et al (2015) Epidemiology of acute kidney injury in critically ill patients: the multinational AKI-EPI study. Intensive Care Med 41:1411-1423. doi:10.1007/s00134-015-3934-7

3. Linder A, Fjell C, Levin A et al (2014) Small acute increases in serum creatinine are associated with decreased long-term survival in the critically ill. Am J Respir Crit Care Med 189:1075-1081. doi:10.1164/ rccm.201311-20970C

4. Coca SG, Yusuf B, Shlipak MG et al (2009) Long-term risk of mortality and other adverse outcomes after acute kidney injury: a systematic review and meta-analysis. Am J Kidney Dis 53:961-973. doi:10.1053/j. ajkd.2008.11.034

5. Chawla LS, Amdur RL, Shaw AD et al (2014) Association between AKI and long-term renal and cardiovascular outcomes in United States veterans. Clin J Am Soc Nephrol 9:448-456. doi:10.2215/CJN.02440213

6. Chawla LS, Amdur RL, Amodeo S et al (2011) The severity of acute kidney injury predicts progression to chronic kidney disease. Kidney Int 79:1361-1369. doi:10.1038/ki.2011.42

7. Schetz M, Gunst J, De Vlieger G, Van den Berghe G (2015) Recovery from $\mathrm{AKI}$ in the critically ill: potential confounders in the evaluation. Intensive Care Med 41:1648-1657. doi:10.1007/s00134-015-3946-3

8. Schetz M, Gunst J, Van den Berghe G (2014) The impact of using estimated GFR versus creatinine clearance on the evaluation of recovery from acute kidney injury in the ICU. Intensive Care Med 40:1709-1717. doi:10.1007/s00134-014-3487-1

9. Mehta RL, McDonald B, Gabbai F et al (2002) Nephrology consultation in acute renal failure: does timing matter? Am J Med 113:456-461

10. Costa e Silva VT, Liaño F, Muriel A et al (2013) Nephrology referral and outcomes in critically ill acute kidney injury patients. PLoS One 8:e70482. doi:10.1371/journal.pone.0070482 
11. Srisawat N, Lawsin L, Uchino S et al (2010) Cost of acute renal replacement therapy in the intensive care unit: results from The Beginning and Ending Supportive Therapy for the Kidney (BEST Kidney) study. Crit Care 14:R46. doi:10.1186/cc8933

12. Truche A-S, Darmon M, Bailly S et al (2016) Continuous renal replacement therapy versus intermittent hemodialysis in intensive care patients: impact on mortality and renal recovery. Intensive Care Med 42:14081417. doi:10.1007/s00134-016-4404-6

13. Rhodes A, Moreno RP, Azoulay E et al (2012) Prospectively defined indicators to improve the safety and quality of care for critically ill patients: a report from the Task Force on Safety and Quality of the European Society of Intensive Care Medicine (ESICM). Intensive Care Med 38:598-605. doi:10.1007/s00134-011-2462-3

14. Harel Z, Wald R, Bargman JM et al (2013) Nephrologist follow-up improves all-cause mortality of severe acute kidney injury survivors. Kidney Int 83:901-908. doi:10.1038/ki.2012.451

15. Kellum JA, Sileanu FE, Bihorac A et al (2016) Recovery after acute kidney injury. Am J Respir Crit Care Med. doi:10.1164/rccm.201604-07990C 